PROCEEDINGS OF THE

AMERICAN MATHEMATICAL SOCIETY

Volume 139, Number 5, May 2011, Pages 1879-1887

S 0002-9939(2010)10580-4

Article electronically published on October 6, 2010

\title{
SOME REMARKS ON CONFIGURATION SPACES
}

\author{
GEORGE RAPTIS
}

(Communicated by Brooke Shipley)

\begin{abstract}
This paper studies the homotopy type of the configuration spaces $F_{n}(X)$ by introducing the idea of configuration spaces of maps. For every map $f: X \rightarrow Y$, the configuration space $F_{n}(f)$ is the space of configurations in $X$ that have distinct images in $Y$. We show that the natural maps $F_{n}(X) \leftarrow$ $F_{n}(f) \rightarrow F_{n}(Y)$ are homotopy equivalences when $f$ is a proper cell-like map between $d$-manifolds. We also show that the best approximation to $X \mapsto$ $F_{n}(X)$ by a homotopy invariant functor is given by the $n$-fold product map.
\end{abstract}

\section{INTRODUCTION}

The space of ordered configurations of $n$ points in $X$ is defined to be the space $F_{n}(X)=\left\{\left(x_{1}, \ldots, x_{n}\right) \in X^{n} \mid x_{i} \neq x_{j}\right.$ if $\left.i \neq j\right\} \subset X^{n}$. The homotopy theory of configuration spaces has been studied extensively in connection with iterated loop spaces [18] and mapping spaces in general [3]. For such purposes, it is necessary to "stabilise" with respect to $n$, i.e., to consider the space of all configurations of points in $X$. In the present note, we will be concerned with the study of the homotopy type of the configuration spaces from the "unstable" point of view.

First, it can be easily seen that the homotopy type of $F_{n}(X)$ is not an invariant of the homotopy type of $X$. This naturally leads to the question of what is the best approximation to the correspondence $X \mapsto F_{n}(X)$ by a homotopy invariant functor. In Section 2, we show that such a homotopical approximation exists and that it is given by the $n$-fold product functor $X \mapsto X^{n}$.

The question of the homotopy invariance of the ordered configuration spaces $F_{n}(X)$ becomes more interesting when one restricts to closed manifolds. Levitt 13 showed that the homotopy type of the loop space $\Omega F_{n}(M)$ of the configuration space of a closed smooth manifold $M$ depends only on the homotopy type of $M$. Aouina and Klein [1] showed that the homotopy type of a certain iterated suspension of the configuration space $F_{n}(M)$ of a closed PL manifold $M$ is also an invariant of the homotopy type of $M$. Moreover, an explicit sufficient number of suspensions is given depending on $n$, the dimension of $M$ and the connectivity of $M$. Both results may be viewed as evidence that the configuration spaces are homotopy invariant, an assertion that was nevertheless disproved by Longoni and Salvatore [14. Longoni and Salvatore [14 discovered an example of a pair of homotopy equivalent lens spaces whose configuration spaces are not homotopy equivalent. The nature of the

Received by the editors April 30, 2010 and, in revised form, May 11, 2010.

2010 Mathematics Subject Classification. Primary 55R80; Secondary 57N99.

Key words and phrases. Configuration spaces, cell-like maps.

(C)2010 American Mathematical Society 
counterexample naturally suggests the question of whether the configuration spaces of closed manifolds are simple-homotopy invariant.

A major source of difficulty in problems related to configuration spaces (or, more generally, spaces of embeddings) arises from the lack of functoriality. This means that, given a map $f: X \rightarrow Y$, there is no natural way of producing a map " $F_{n}(f): F_{n}(X) \rightarrow F_{n}(Y)$ " unless $f$ is injective. But injectivity is not a suitable property for homotopy-theoretic considerations. A quite drastic answer to this problem is to consider the relative configuration spaces

$$
F_{n}(f)=\left\{\left(x_{1}, \cdots, x_{n}\right) \in F_{n}(X) \mid f\left(x_{i}\right) \neq f\left(x_{j}\right) \text { if } i \neq j\right\}
$$

associated with a map $f: X \rightarrow Y$, together with the natural zigzag of maps

$$
F_{n}(X) \hookleftarrow F_{n}(f) \stackrel{f_{\text {* }}^{\rightarrow}}{\rightarrow} F_{n}(Y) .
$$

In Section 3, we study the functoriality properties of these configuration spaces of maps. In Section 4, we give sufficient conditions for the maps in the zigzag diagram to be weak homotopy equivalences. More specifically, we show that this is always the case when $f$ is a proper cell-like map between topological manifolds of the same dimension.

Terminology. The term space will always refer to a compactly generated topological space. The term manifold will always refer to a connected topological manifold without boundary.

\section{The homotopical approximation to $X \mapsto F_{n}(X)$}

Let $\mathcal{T}$ op denote the category of compactly generated spaces and continuous maps, and let $\mathcal{T} o p_{<}$be the subcategory of injective continuous maps. Consider the functor $F_{n}: \mathcal{T}_{o p_{<}} \rightarrow \mathcal{T}$ op that is defined on objects by sending a space $X$ to the space of configurations $F_{n}(X)$. It is easy to see that $F_{n}$ does not preserve the weak homotopy equivalences. For a trivial example that shows how badly this fails, note that for $n>1, F_{n}\left(\mathbb{R}^{2}\right)$ is always connected and has a non-trivial fundamental group, but $F_{n}(\mathbb{R})$ is not connected and its components are contractible.

A homotopical approximation to $F_{n}$ is a pair $(G, \eta)$ where $G: \mathcal{T}$ op $\rightarrow \mathcal{T}$ op is a functor that preserves the weak homotopy equivalences, together with a natural transformation $\eta: F_{n} \rightarrow G$ of functors from $\mathcal{T} o p_{<}$to $\mathcal{T} o p$, such that for every other such pair $\left(G^{\prime}, \eta^{\prime}\right)$ there is a natural transformation up to homotopy $\gamma: G \rightarrow G^{\prime}$ that is unique up to homotopy and $\eta^{\prime} \simeq \gamma \circ \eta$. The homotopical approximation can be thought of as the total right derived functor of $F_{n}$ up to the peculiarity of the domain of definition of $F_{n}$. Furthermore, it is clear that a homotopical approximation, if it exists, is unique up to a canonical homotopy equivalence.

A homotopical approximation to $F_{n}$ exists, and it is given by the $n$-fold product functor $X \mapsto X^{n}$. The proof of this assertion rests on the following trick.

Lemma 2.1. For every space $X$ and $n \geqslant 1$, there is a homotopy equivalence $F_{n}\left(S^{\infty} \times X\right) \simeq X^{n}$.

Proof. Let $\underline{s}=\left(s_{1}, \cdots, s_{n}\right) \in F_{n}\left(S^{\infty}\right)$. There is an inclusion $i: X^{n} \rightarrow F_{n}\left(X \times S^{\infty}\right)$, $\left(x_{1}, \cdots, x_{n}\right) \mapsto\left(\left(x_{1}, s_{1}\right), \cdots,\left(x_{n}, s_{n}\right)\right)$, and a projection $p: F_{n}\left(X \times S^{\infty}\right) \rightarrow X^{n}$. Clearly $p i=1$. Also, there is a homotopy $i p \simeq 1$, because $\left(\left(S^{\infty}\right)^{n}, F_{n}\left(S^{\infty}\right)\right) \simeq *$. The result follows.

Let $\mathbf{n}$ denote the discrete space with $n$ points. 
Theorem 2.2. The $n$-fold product functor $\operatorname{Map}(\mathbf{n},-): \mathcal{T}$ op $\rightarrow \mathcal{T}$ op is the homotopical approximation to $F_{n}$.

Proof. The functor $\operatorname{Map}(\mathbf{n},-)$ is homotopy invariant, and there are natural inclusion maps $\eta_{X}: F_{n}(X) \rightarrow X^{n}$ for every space $X$. Therefore it suffices to show that the universal property is satisfied. Let $\left(G, \eta^{\prime}\right)$ be another pair with these properties. For every $X$, the inclusion $i_{X}: X \rightarrow X \times S^{\infty}$ is a homotopy equivalence, and the projection $p_{X}$ is a homotopy inverse. Thus the map $G\left(i_{X}\right): G(X) \rightarrow G\left(X \times S^{\infty}\right)$ is a homotopy equivalence, and there is a natural choice $G\left(p_{X}\right)$ of a homotopy inverse. By Lemma 2.1, there is a homotopy equivalence $X^{n} \stackrel{\simeq}{\rightarrow} F_{n}\left(X \times S^{\infty}\right)$. Thus there is a map

$$
\gamma_{X}: X^{n} \stackrel{\widetilde{\Im}}{\rightarrow} F_{n}\left(X \times S^{\infty}\right) \stackrel{\eta_{X \times S^{\infty}}^{\prime}}{\longrightarrow} G\left(X \times S^{\infty}\right) \stackrel{G\left(p_{X}\right)}{\longrightarrow} G(X)
$$

such that $\eta_{X}^{\prime} \simeq \gamma_{X} \circ \eta_{X}$ for every $X$, as required.

Furthermore, this map is natural up to homotopy. Let $f: X \rightarrow Y$ and $M_{f}$ be the mapping cylinder. Then there is a diagram

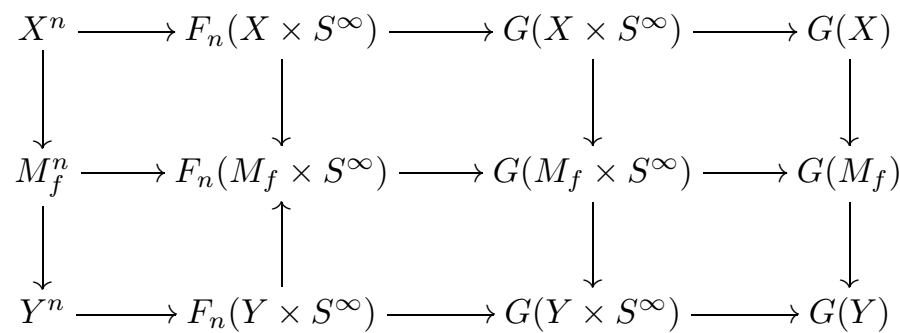

that commutes up to homotopy, and the "wrong"-way vertical arrow is a homotopy equivalence.

Finally, the map $\gamma_{X}: X^{n} \rightarrow G(X)$ is unique up to homotopy because for every other such map $\delta_{X}: X^{n} \rightarrow G(X)$, there is a diagram

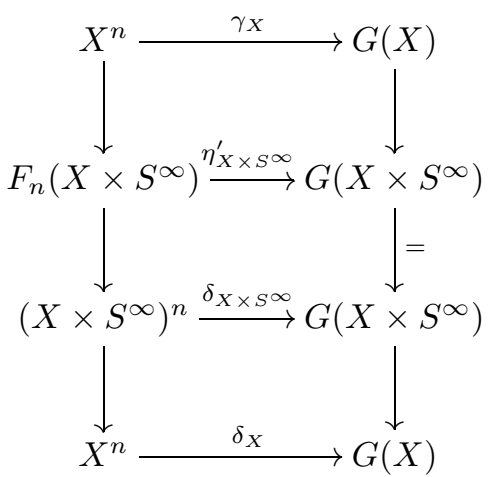

that commutes up to homotopy and the vertical composites are identities.

\section{Configuration SPACES OF MAPS}

Definition 3.1. Let $f: X \rightarrow Y$ be a map and $n \geqslant 1$. The configuration space $F_{n}(f)$ is defined to be $F_{n}(f):=\left\{\left(x_{1}, \cdots, x_{n}\right) \in X^{n} \mid f\left(x_{i}\right) \neq f\left(x_{j}\right)\right.$ if $\left.i \neq j\right\} \subset$ $F_{n}(X)$. 
There is a map $f_{\star}: F_{n}(f) \rightarrow F_{n}(Y)$ that takes a configuration of points in $X$ to its image in $Y$. In fact, $F_{n}(f)$ is exactly the largest open subspace of $F_{n}(X)$ for which this map is well-defined. Note that for any injective map $f: X \rightarrow Y$, we have $F_{n}(f)=F_{n}(X)$, and in particular, $F_{n}(X)=F_{n}\left(1_{X}\right)$.

For every space $Z$, there is a functor

$$
\mathcal{F}_{n}: \mathcal{T} \text { op } \downarrow Z \rightarrow \mathcal{T} \text { op } \downarrow F_{n}(Z)
$$

that takes an object $(f: X \rightarrow Z)$ to $\left(f_{\star}: F_{n}(f) \rightarrow F_{n}(Z)\right)$ and a morphism $u:(X, f) \rightarrow(Y, g)$ to the morphism $u_{\star}:\left(F_{n}(f), f_{\star}\right) \rightarrow\left(F_{n}(g), g_{\star}\right)$. Recall that the objects of the category $\mathcal{T}$ op $\downarrow Z$ are the pairs $(X, f)$ where $X$ is a space and $f: X \rightarrow Z$ is a map, and a morphism from $(X, f)$ to $(Y, g)$ is given by a map $u: X \rightarrow Y$ such that $f=g u$.

Proposition 3.1. There is an adjunction

$$
\mathcal{T}_{n}: \mathcal{T}_{o p} \downarrow F_{n}(Z) \rightleftarrows \mathcal{T} o p \downarrow Z: \mathcal{F}_{n} .
$$

Moreover, the functor $\mathcal{F}_{n}$ is topological, i.e. for any $f, g \in \mathcal{T}$ op $\downarrow Z$ the map

$$
\operatorname{Map}_{\mathcal{T} o p \downarrow Z}(f, g) \rightarrow \operatorname{Map}_{\mathcal{T} o p \downarrow F_{n}(Z)}\left(f_{\star}, g_{\star}\right)
$$

is continuous.

Proof. The functor $\mathcal{T}_{n}: \mathcal{T} o p \downarrow F_{n}(Z) \rightarrow \mathcal{T}$ op $\downarrow Z$ sends an object $(X, f)$ to $\left(X \times[n], f^{\star}\right)$ where $f^{\star}(x, k)=f(x)_{k}$. On morphisms, it takes $u:(X, f) \rightarrow(Y, g)$ to $u \times 1_{[n]}:\left(X \times[n], f^{\star}\right) \rightarrow\left(Y \times[n], g^{\star}\right)$.

Let $u: \mathcal{T}_{n}(X, f)=\left(X \times[n], f^{\star}\right) \rightarrow(Y, g)$ be a morphism in $\mathcal{T}$ op $\downarrow Z$. It defines a morphism $\theta(u):(X, f) \rightarrow\left(F_{n}(g), g_{\star}\right)$ by $\theta(u)(x)=(u(x, 1), \cdots, u(x, n))$.

Conversely, a morphism $u^{\prime}:(X, f) \rightarrow \mathcal{F}_{n}(Y, g)$ in $\mathcal{T}$ op $\downarrow F_{n}(Z)$ defines a morphism $\phi\left(u^{\prime}\right):\left(X \times[n], f^{\star}\right) \rightarrow(Y, g)$ in $\mathcal{T} o p \downarrow Z$ by $\phi\left(u^{\prime}\right)(x, k)=u^{\prime}(x)_{k}$.

Clearly, $\theta$ and $\phi$ define a pair of natural inverse bijections

$$
\theta: \operatorname{Hom}_{\mathcal{T} o p \downarrow Z}\left(\mathcal{T}_{n}(X, f),(Y, g)\right) \rightleftarrows H m_{\mathcal{T} o p \downarrow F_{n}(Z)}\left((X, f), \mathcal{F}_{n}(Y, g)\right): \phi
$$

and therefore $\mathcal{T}_{n}$ is left adjoint to $\mathcal{F}_{n}$.

For $f: X \rightarrow Z$ and $g: Y \rightarrow Z, \operatorname{Map}_{\mathcal{T} o p \downarrow Z}(f, g)$ is defined as a subspace of $\operatorname{Map}(X, Y)$. The map $\operatorname{Map}_{\mathcal{T} o p \downarrow Z}(f, g) \rightarrow \operatorname{Map}_{\mathcal{T} o p \downarrow F_{n}(Z)}\left(f_{\star}, g_{\star}\right)$ is just a restriction of the map $\operatorname{Map}(X, Y) \rightarrow \operatorname{Map}\left(X^{n}, Y^{n}\right) \rightarrow \operatorname{Map}\left(F_{n}(f), Y^{n}\right)$, therefore it is continuous.

The adjunction is compatible with the homotopy theories of the associated categories in a sense that comes from the theory of model categories [10. A morphism $u:(X, f) \rightarrow(Y, g)$ in $\mathcal{T}$ op $\downarrow Z$ is called a weak equivalence (resp. fibration) if the corresponding map $u: X \rightarrow Y$ is a weak homotopy equivalence (resp. Serre fibration). These classes of morphisms define a model structure on $\mathcal{T}$ op $\downarrow Z$ (see [9. Theorem 7.6.5] and [10, Theorem 2.4.25]). The cofibrations are exactly the maps that have the left lifting property with respect to every map that is a fibration and a weak equivalence. A morphism which is both a (co)fibration and a weak equivalence is called a trivial (co)fibration.

Proposition 3.2. The adjunction $\mathcal{T}_{n}: \mathcal{T}$ op $\downarrow F_{n}(Z) \rightleftarrows \mathcal{T}$ op $\downarrow Z: \mathcal{F}_{n}$ is a Quillen adjunction of model categories.

Proof. The classes of cofibrations and trivial cofibrations are clearly closed under coproducts. Therefore $\mathcal{T}_{n}$ is a left Quillen functor. 
It follows that $\mathcal{F}_{n}$ preserves fibrations and trivial fibrations. If $p: E \rightarrow B$ is a trivial fibration, then $F_{n}(p) \rightarrow F_{n}(B)$ is also a trivial fibration, so in particular, a weak homotopy equivalence. Also, if $f: X \rightarrow Z$ is a map and $p: M_{f} \rightarrow Z$ is the usual deformation retraction from the mapping cylinder of $f$ onto $Z$, then the inclusion $i: Z \rightarrow M_{f}$ is a homotopy equivalence over $Z$. Hence $i_{\star}: F_{n}\left(1_{Z}\right) \rightarrow F_{n}(p)$ is a homotopy equivalence with homotopy inverse $p_{\star}: F_{n}(p) \rightarrow F_{n}(Z)$.

Given a map $f: X \rightarrow Z$, the homotopy type of $F_{n}(f)$ may differ dramatically from that of $F_{n}(X)$ or $F_{n}(Z)$. Moreover, the homotopy type of $F_{n}(f)$ is not an invariant of the homotopy class of the map $f$. We will be interested in a class of maps $f$ for which the map $f_{\star}: F_{n}(f) \rightarrow F_{n}(Z)$ and the inclusion $F_{n}(f) \rightarrow F_{n}(X)$ are weak homotopy equivalences.

\section{Configuration spaces of manifolds}

Let $M$ be a topological $d$-dimensional manifold. For every $n \geqslant 1$, the projection $F_{n+1}(M) \rightarrow F_{n}(M)$ of the first $n$ coordinates is a fiber bundle whose fiber at $\left(m_{1}, \cdots, m_{n}\right)$ is $M-\left\{m_{1}, \cdots, m_{n}\right\}$; see [8]. Following [13, we write $M_{-n}$ for the manifold $M$ with $n$ distinct points removed. The homeomorphism type of $M_{-n}$ is independent of the choice of $n$ points. Moreover, if $f: M \rightarrow N$ is a homotopy equivalence between $d$-dimensional manifolds, then there is a homotopy equivalence $f_{-n}: M_{-n} \rightarrow N_{-n}$ that makes the diagram

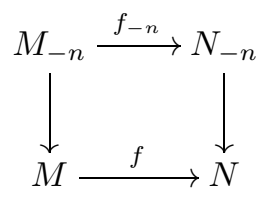

commute up to homotopy. In detail, this can be seen as follows: first, since $M_{-n}=$ $\left(M_{-(n-1)}\right)_{-1}$, it suffices to consider only the case $n=1$. There are cofiber sequences

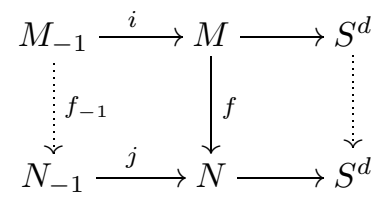

where the inclusions $i$ and $j$ are $(d-1)$-connected maps. Since $M_{-1}$ has trivial cohomology in degrees greater than $d-1$, it follows that $M_{-1} \rightarrow N$ factors up to homotopy through a map $f_{-1}: M_{-1} \rightarrow N_{-1}$ that is a homotopy equivalence.

Hence the homotopy type of $M_{-n}$ is an invariant of the homotopy type of $M$ as a $d$-dimensional manifold, so the spaces $F_{n}(M)$ can be viewed as a succession of total spaces of fiber bundles whose fiber at each stage is determined up to homotopy equivalence by $M$.

Proposition 4.1. Let $f: M \rightarrow N$ be a homotopy equivalence between d-dimensional manifolds and $m \geqslant 1$. Then the inclusion $F_{n}(f) \rightarrow F_{n}(M)$ is a homotopy equivalence for all $1 \leqslant n \leqslant m$ if and only if the map $f_{\star}: F_{n}(f) \rightarrow F_{n}(N)$ is a homotopy equivalence for all $1 \leqslant n \leqslant m$. 
Proof. Consider the commutative diagram

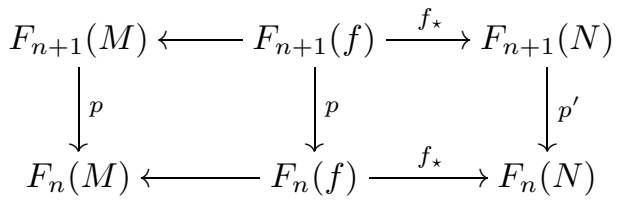

and let $X_{n}$ denote the homotopy fiber of $F_{n+1}(f) \rightarrow F_{n}(f)$. There are natural unique maps up to homotopy $s_{n}: X_{n} \rightarrow M_{-n}$ and $t_{n}: X_{n} \rightarrow N_{-n}$. The map $t_{n}$ is given by the top row of the diagram

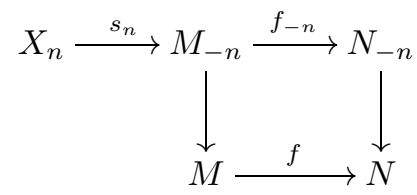

where $f_{-n}$ is a homotopy equivalence that makes the square commute up to homotopy. Therefore the map $s_{n}$ is a homotopy equivalence if and only if $t_{n}$ is as well. If $F_{n}(f) \rightarrow F_{n}(M)$ is a homotopy equivalence for $1 \leqslant n \leqslant m$, then $s_{n}: X_{n} \rightarrow M_{-n}$ is a homotopy equivalence for $1 \leqslant n \leqslant m-1$. Therefore $t_{n}$ is also a homotopy equivalence for $1 \leqslant n \leqslant m-1$. By passing to the long exact sequences of homotopy groups and Whitehead's theorem, it follows inductively that $F_{n}(f) \rightarrow F_{n}(N)$ is a homotopy equivalence for all $1 \leqslant n \leqslant m$. The converse statement is similar.

The assertion that the inclusions $F_{n}(f) \rightarrow F_{n}(M)$ are homotopy equivalences for all $n \geqslant 1$ is very strong. It seems sensible to think that it should relate to the assertion that the point-preimages of $f$ are homotopically trivial.

Definition 4.1. (a) A subset $K \subset M$ of a $d$-dimensional manifold $M$ is called cellular in $M$ if it is the intersection of a sequence $B_{1}, B_{2}, \cdots$ of closed $d$-cells in $M$ such that $B_{i} \subset \operatorname{Int}\left(B_{i+1}\right)$.

(b) A space $K$ is called cell-like if there is a $d$-dimensional manifold $M$ and an embedding $\phi: K \rightarrow M$ such that $\phi(K)$ is cellular in $M$.

The concept of cellularity was introduced in the work of Brown 4. Note that cellularity depends on the embedding of $K$ rather than just $K$ itself. The wider concept of a cell-like space was introduced by Lacher [11.

Theorem 4.2. Let $X$ be a non-empty finite-dimensional compact metric space. Then the following are equivalent:

(i) $X$ is cell-like.

(ii) $X$ has the shape (or Čech homotopy type) of a point.

(iii) There exists an embedding of $X$ in an ENR $M$ such that the following property holds:

$U V^{\infty}$ : for every neighborhood $U$ of $X$ in $M$, there exists a neighborhood $V$ such that $X \subset V \subset U$ and the inclusion $V \rightarrow U$ is nullhomotopic.

(iv) For any embedding of $X$ into an ANR property, $U V^{\infty}$ holds.

Proof. See [11, Theorem 1], [12, Theorem 1.1]. 
Definition 4.2. (a) A map $f: M \rightarrow Y$ from a manifold $M$ is called cellular if for every $y \in Y$ the inverse image $f^{-1}(y)$ is cellular in $M$.

(b) A map $f: X \rightarrow Y$ is called cell-like if for every $y \in Y$ the inverse image $f^{-1}(y)$ is cell-like.

Cell-like maps have been studied by Lacher [12. For a nice survey on the subject including a comprehensive bibliography, see [15. Note that cell-like maps are necessarily surjective. They generalise the notion of simple (or contractible) maps. Recall that a PL map $f: K \rightarrow L$ between finite polyhedra is called simple if for every $y \in L$, the inverse image $f^{-1}(y)$ is contractible. Every cell-like map between finite CW complexes is a simple-homotopy equivalence [5], and therefore the class of cell-like maps provides one with a useful rule for detecting simple-homotopy equivalences (see also [6]). In general, a simple-homotopy equivalence can be written as a zigzag of cell-like maps. See [7] for background in simple-homotopy theory. The following useful result is proved in [12].

Theorem 4.3. Let $X$ and $Y$ be ENRs and let $f: X \rightarrow Y$ be a proper surjective map. Then $f$ is cell-like if and only if for every open subset $U \subseteq Y,\left.f\right|_{f^{-1}(U)}$ : $f^{-1}(U) \rightarrow U$ is a proper homotopy equivalence. In particular, if $f$ is a proper cell-like map, then it is a proper homotopy equivalence.

Proof. See [12, Theorem 1.2].

Proposition 4.4. Let $f: X \rightarrow Y$ be a proper cell-like map between ENRs. Then $f_{\star}: F_{n}(f) \rightarrow F_{n}(Y)$ is proper and cell-like for all $n \geqslant 1$. In particular, $f_{\star}$ is a proper homotopy equivalence.

Proof. The inverse image of a point $\left(y_{1}, \cdots, y_{n}\right) \in F_{n}(Y)$ is a product of celllike spaces, and therefore it is also cell-like by [12, Theorem 1.5]. In order to show that $f_{\star}$ is proper, it suffices to show that every point $\left(y_{1}, \cdots, y_{n}\right) \in F_{n}(Y)$ has a neighborhood whose inverse image has compact closure. We can find open neighborhoods $U_{i}$ in $Y$ such that $y_{i} \in U_{i}, \bar{U}_{i}$ is compact for all $i$ and $\bar{U}_{i} \cap \bar{U}_{j}=\varnothing$ for all $i \neq j$. The inverse image of $U_{1} \times \cdots \times U_{n}$ under $f_{\star}$ is $f^{-1}\left(U_{1}\right) \times \cdots \times f^{-1}\left(U_{n}\right)$. Each $f^{-1}\left(U_{i}\right)$ has compact closure because $f^{-1}\left(\bar{U}_{i}\right)$ is compact and $\overline{f^{-1}\left(U_{i}\right)} \subset f^{-1}\left(\bar{U}_{i}\right)$. The result follows.

Theorem 4.5. Let $f: M \rightarrow N$ be a proper cell-like map between d-manifolds. Then the natural maps $F_{n}(M) \leftarrow F_{n}(f) \rightarrow F_{n}(N)$ are homotopy equivalences for all $n \geqslant 1$.

Proof. This follows immediately from Proposition 4.4 and Proposition 4.1 .

Remark 1. In the situation of the theorem, note that the projections $F_{k+1}(f) \rightarrow$ $F_{k}(f)$ are quasifibrations for all $k$. Furthermore, let $E=\left\{\left(m_{1}, \cdots, m_{k}, n\right) \in\right.$ $F_{k}(f) \times N \mid n \neq f\left(m_{j}\right)$ for all $\left.j\right\}$ be the total space of the bundle over $F_{k}(f)$ pulled back from $F_{k+1}(N) \rightarrow F_{k}(N)$ along $f_{\star}: F_{k}(f) \rightarrow F_{k}(N)$, and let $g: F_{k+1}(f) \rightarrow E$ be the canonically induced map. Then $g$ is a proper cell-like map.

The limit of a sequence of homeomorphisms between homeomorphic manifolds in the space of all continuous maps is always a cellular map. It turns out that the converse is also true.

Theorem 4.6 (Cell-Like Approximation Theorem). For every proper cell-like map $f: M \rightarrow N$ between d-manifolds and every continuous map $\epsilon: M \rightarrow(0, \infty)$, there 
is a homeomorphism $h: M \rightarrow N$ such that $d(f(x), h(x))<\epsilon(x)$ for all $x \in M$. [If $d=3$, one must assume that $f$ is cellular].

Proof. This was proved by Siebenmann $[19$ in all dimensions $d \neq 4$. The result in dimension 4 was proved by Quinn [17]. The low-dimensional cases $d \leqslant 3$ were also studied earlier by Armentrout [2, Youngs [20] and Roberts and Steenrod [16].

Corollary 4.7. Let $f: M \rightarrow N$ be a proper cell-like map between d-manifolds. Then the spaces $F_{n}(M), F_{n}(f)$ and $F_{n}(N)$ are homeomorphic for all $n \geqslant 1$. [If $d=3$, assume that $f$ is cellular $]$.

Proof. By Theorem 4.6, there is a homeomorphism $h: M \rightarrow N$. It follows that $F_{n}(M)$ and $F_{n}(N)$ are homeomorphic. Note that $F_{n}(f)$ is a submanifold of $F_{n}(M)$ of the same dimension. Then, by Proposition 4.4 and Theorem 4.6, it follows that $F_{n}(f)$ and $F_{n}(N)$ are also homeomorphic.

\section{ACKNowledgements}

The author would like to thank his D.Phil. supervisor, Ulrike Tillmann, for her support and advice. He would also like to gratefully acknowledge the financial support of an EPSRC Studentship and a scholarship from the Alexander S. Onassis Public Benefit Foundation.

\section{REFERENCES}

1. M. Aouina, J. R. Klein, On the homotopy invariance of configuration spaces, Algebr. Geom. Topol. 4 (2004), 813-827 (electronic). MR 2100681 (2005k:55021)

2. S. Armentrout, Cellular decompositions of 3-manifolds that yield 3-manifolds, Mem. Amer. Math. Soc., No. 107, American Mathematical Society, 1971. MR0413104 (54:1225)

3. C.-F. Bödigheimer, Stable splittings of mapping spaces, Algebraic topology (Seattle, Wash., 1985), Lecture Notes in Math., No. 1286, pp. 174-187, Springer, Berlin, 1987. MR922926 (89c:55011)

4. M. Brown, A proof of the generalized Schoenflies theorem, Bull. Amer. Math. Soc. 66 (1960), 74-76. MR0117695 (22:8470b)

5. T. A. Chapman, Cell-like mappings, Algebraic and geometrical methods in topology, Lecture Notes in Math., Vol. 428, pp. 230-240, Springer, Berlin, 1974. MR0383423 (52:4304)

6. T. A. Chapman, Homotopy conditions which detect simple homotopy equivalences, Pacific J. Math. 80 (1979), no. 1, 13-46. MR.534693 (81f:57011)

7. M. M. Cohen, A course in simple-homotopy theory. Graduate Texts in Mathematics, Vol. 10, Springer-Verlag, New York-Berlin, 1973. MR0362320 (50:14762)

8. E. Fadell, L. Neuwirth, Configuration spaces, Math. Scand. 10 (1962), 111-118. MR0141126 $(25: 4537)$

9. P. S. Hirschhorn, Model categories and their localizations. Mathematical Surveys and Monographs, Vol. 99, American Mathematical Society, 2003. MR.1944041 (2003j:18018)

10. M. Hovey, Model Categories. Mathematical Surveys and Monographs, Vol. 63, American Mathematical Society, 1999. MR.1650134 (99h:55031)

11. R. C. Lacher, Cell-like mappings of ANRs, Bull. Amer. Math. Soc. 74 (1968), 933-935. MR.0244963 (39:6276)

12. R. C. Lacher, Cell-like mappings. I, Pacific J. Math. 30 (1969), 717-731. MR0251714(40:4941)

13. N. Levitt, Spaces of arcs and configuration spaces of manifolds, Topology 34 (1995), no. 1, 217-230. MR.1308497 (95k:55015)

14. R. Longoni, P. Salvatore, Configuration spaces are not homotopy invariant, Topology 44 (2005), no. 2, 375-380. MR2114713 (2005k:55024)

15. W. J. R. Mitchell, D. Repovš, The topology of cell-like mappings, Conference on Differential Geometry and Topology, Rend. Sem. Fac. Sci. Univ. Cagliari 58 (1988), 265-300. MR 1122860 (92f:54012) 
16. J. H. Roberts, N. E. Steenrod, Monotone transformations of two-dimensional manifolds, Ann. of Math. (2) 39 (1938), no. 4, 851-862. MR.1503441

17. F. Quinn, Ends of maps. III: Dimensions 4 and 5, J. Differential Geom. 17 (1982), no. 3, 503-521. MR679069 (84j:57012)

18. G. Segal, Configuration-spaces and iterated loop-spaces, Invent. Math. 21 (1973), 213-221. MR 0331377 (48:9710)

19. L. C. Siebenmann, Approximating cellular maps by homeomorphisms, Topology 11 (1972), 271-294. MR0295365 (45:4431)

20. J. W. T. Youngs, Homeomorphic approximations to monotone mappings, Duke Math. J. 15 (1948), 87-94. MR0024623(9:524a)

Institut für Mathematik, Universität Osnabrück, Albrechtstrasse 28A, 49069 OsnaBRÜCK, GERMANY

E-mail address: graptis@mathematik.uni-osnabrueck.de 